\title{
Diagnóstico molecular de tuberculosis extrapulmonar y sensibilidad a rifampicina con un método automatizado en tiempo real
}

\author{
Adrián Peñata', Richard Salazar², Tatiana Castaño ${ }^{1}$, Julián Bustamante' ${ }^{1}$, Sigifredo Ospina ${ }^{1}$ \\ 1 Unidad de Biología Molecular e Inmunogénetica de Trasplantes, Departamento de Microbiología, Hospital \\ Universitario San Vicente Fundación, Medellín, Colombia \\ 2 Escuela de Microbiología, Universidad de Antioquia, Medellín, Colombia
}

Introducción. La tuberculosis continúa siendo un problema de salud pública mundial, y la forma extrapulmonar representa entre 10 y $20 \%$ del total de casos en personas inmunocompetentes, porcentaje que se incrementa en los portadores del virus de la inmunodeficiencia humana (HIV). Su diagnóstico es difícil con los métodos convencionales por la naturaleza paucibacilar de las muestras. La prueba Xpert ${ }^{\oplus}$ MTB/RIF ha significado un importante avance en la detección molecular de Mycobacterium tuberculosis y hoy se utiliza en una variedad de muestras clínicas obtenidas de sitios diferentes a las vías respiratorias.

Objetivo. Determinar la utilidad de la prueba Xpert ${ }^{\circledR}$ MTB/RIF en la detección de $M$. tuberculosis y la sensibilidad a la rifampicina en pacientes con sospecha de tuberculosis extrapulmonar, atendidos en el Hospital Universitario de San Vicente Fundación de Medellín entre 2013 y 2014.

Materiales y métodos. Se hizo un estudio descriptivo de corte transversal retrospectivo y prospectivo en 372 muestras consecutivas provenientes de 301 pacientes con sospecha de tuberculosis extrapulmonar, las cuales se analizaron mediante baciloscopia, cultivo en medio de Ogawa-Kudoh y prueba molecular Xpert ${ }^{\circledR}$ MTB/RIF.

Resultados. De los pacientes estudiados, 182 eran hombres (60\%) y la condición de base más frecuentemente diagnosticada fue la infección por HIV. Al tomar como referencia el cultivo, la sensibilidad y la especificidad general de la prueba molecular fueron de $94 \%\left(\mathrm{IC}_{95 \%} 83-100\right)$ y $97 \%\left(\mathrm{IC}_{95 \%}\right.$ 95-99), respectivamente, y para la baciloscopia, fueron de $38,71 \%\left({ }^{2} \mathrm{C}_{95 \%}\right.$ 19-57) y $100 \%$ (IC $\left.{ }_{95 \%} 99-100\right)$, respectivamente. En el análisis estratificado por muestras se encontraron sensibilidades mayores de $75 \%$; 37 aislamientos fueron sensibles a la rifampicina y uno fue resistente.

Conclusión. La prueba Xpert ${ }^{\circledR}$ MTB/RIF tuvo un buen desempeño en muestras de diferentes tejidos y líquidos, y constituye un avance significativo como apoyo para el diagnóstico de la tuberculosis extrapulmonar en términos de tiempo y porcentaje de positividad.

Palabras clave: Mycobacterium tuberculosis, tuberculosis miliar, reacción en cadena de la polimerasa, ADN, rifampicina.

doi: http://dx.doi.org/10.7705/biomedica.v36i3.3088 Molecular diagnosis of extrapulmonary tuberculosis and sensitivity to rifampicin with an
automated real-time method

Introduction: Tuberculosis continues to be a global public health problem, the extrapulmonary form being estimated to occur in $10-20 \%$ of immunocompetent individuals, increasing in patients who are carriers of the human immunodeficiency virus (HIV); its diagnosis is difficult with conventional methods due to the paucibacillary nature of samples. The Xpert ${ }^{\oplus}$ MTB/RIF test represents an important development in the molecular detection of Mycobacterium tuberculosis and has been used with a variety of non-respiratory clinical samples.

Objective: To determine the effectiveness of Xpert ${ }^{\circledR}$ MTB/RIF in the detection of $M$. tuberculosis and sensitivity to rifampicin in patients with suspected extrapulmonary tuberculosis attending Hospital Universitario de San Vicente Fundación in Medellín in 2013-2014.

Materials and methods: This was a descriptive, cross-sectional ambispective study of 372 consecutive samples from 301 patients with suspected extrapulmonary tuberculosis, who were subjected to bacilloscopy, followed by culture in Ogawa Kudoh and the Xpert ${ }^{\circledast}$ MTB/RIF molecular test.

\section{Contribución de los autores:}

Adrián Peñata: diseño del estudio, análisis e interpretación de los resultados y redacción del manuscrito

Richard Salazar, Tatiana Castaño y Julián Bustamante: procesamiento de las muestras y recopilación de la información

Sigifredo Ospina: gestión del proyecto, análisis y redacción del manuscrito

Todos los autores participaron en la revisión de la bibliografía, la discusión y la aprobación del manuscrito. 
Results: The most frequent base diagnosis $(60 \%)$ for the 182 patients was infection with HIV. Using the culture as reference, the sensitivity and general specificity of the molecular test was $94 \%(95 \%$ Cl: $83-100)$ and $97 \%$ (95\% Cl: 95-99), respectively; for bacilloscopy it was $38.71(95 \% \mathrm{Cl}: 19-57)$ and $100 \%$ (95\% Cl: $99-100)$, respectively. Sensitivities higher than $75 \%$ were found in analyses stratified by samples. Thirty-seven of the isolates were sensitive and one resistant to rifampicin.

Conclusion: Xpert ${ }^{\oplus}$ MTB/RIF performed well in samples from different tissues and liquids, representing a significant advance in support of extrapulmonary tuberculosis diagnosis in terms of time and percentage positivity.

Key words: Mycobacterium tuberculosis, tuberculosis, miliary, polymerase chain reaction, DNA, rifampin. doi: http://dx.doi.org/10.7705/biomedica.v36i3.3088

La tuberculosis es la segunda causa de muerte por enfermedades infecciosas alrededor del mundo. Se ha estimado que una tercera parte de la población está infectada, por lo que continúa siendo una amenaza grave para la salud pública (1). En el 2014 se presentaron alrededor de 1,5 millones de muertes relacionadas con esta enfermedad, y la infección concomitante con el virus de la inmunodeficiencia humana (HIV) aportó 0,4 millones a esta cifra, en tanto que las personas sin el virus fueron 1,1 millones. Por otro lado, en ese año se estimó que cerca de 9,6 millones de personas en el planeta adquirieron tuberculosis, pero los casos nuevos reportados a la Organización Mundial de la Salud (OMS) fueron seis millones, lo que significa que $37 \%$ de ellos no fue diagnosticado o no se informó. La misma situación se presentó con la tuberculosis multirresistente, pues de los 480.000 casos estimados, solo se detectaron y reportaron 123.000 (1). En 2013 se notificaron en Colombia 10.849 casos de tuberculosis, es decir, 23,02 casos por cada 100.000 habitantes, cifra que se ha mantenido estable en los últimos años, lo que sitúa al país como de incidencia intermedia a nivel mundial (2).

La infección por el HIV ha cambiado la epidemiología de la tuberculosis aumentando el riesgo de las formas no pulmonares de la enfermedad (3). La tuberculosis extrapulmonar se presenta como consecuencia de la diseminación de Mycobacterium tuberculosis a otros órganos por los vasos linfáticos o el torrente circulatorio. Entre los órganos más afectados están las

\footnotetext{
Correspondencia:

Carlos Adrián Peñata, Unidad de Biología Molecular e Inmunogénetica de Trasplantes, Hospital Universitario San Vicente Fundación, Calle 64 № 51D-154, Medellín, Colombia Teléfono: (574) 444 1333; extensión: 2567-3106 carlosadrianpb@gmail.com

Recibido: 02/10/15; aceptado: 27/01/16
}

meninges, la pleura, la piel, los ganglios linfáticos, el abdomen, el aparato genitourinario, los riñones, la piel, las articulaciones y los huesos $(4,5)$.

Aunque este tipo de tuberculosis supone entre 10 y $20 \%$ del total de los casos en personas inmunocompetentes, dichos porcentajes se incrementan hasta en $60 \%$ en pacientes con HIV (6). En esta forma de la enfermedad, la baciloscopia generalmente es negativa, la capacidad de contagio es baja y su detección temprana supone un desafío, ya que en ocasiones cursa con pocos síntomas o puede confundirse con otras condiciones; además, se registran retrasos en el diagnóstico debido a que es una manifestación clínica atípica de la enfermedad y a que la carga bacteriana es escasa (6).

Los casos de tuberculosis extrapulmonar han aumentado en las últimas décadas: en la década del 60 , se estimaba en $1,2 \%$ en Asia y en $8 \%$ en América, cifras que llegaron a 6,7\% en Asia y a $17 \%$ en América, durante la década de los 90 (7). En Colombia, se han encontrado reportes de las secretarías de salud con porcentajes de hasta $37,5 \%$, lo que confirma que se trata de una forma importante de presentación de la enfermedad que exigiría su inclusión entre los diagnósticos diferenciales en los casos sospechosos (8).

Como es sabido, el diagnóstico rápido de la tuberculosis es fundamental para su tratamiento y el control de su propagación (2). En cuanto a las formas extrapulmonares, si bien su capacidad de contagio es poca, tienden a ser más agresivas 0 a generar secuelas que pueden limitar de manera significativa la calidad de vida del paciente (8).

Los métodos actuales de diagnóstico de la tuberculosis se basan en la utilización combinada de la baciloscopia y el aislamiento del microorganismo en el cultivo, pero ambos presentan inconvenientes: primero, por su sensibilidad, la cual oscila entre 40 y $60 \%$, y segundo, por el tiempo requerido 
para obtener resultados, pues una adecuada identificación llega a tardar entre seis y ocho semanas (5). En las formas extrapulmonares de la enfermedad, además, estas dificultades son más críticas, ya que este tipo de muestras son paucibacilares (8).

En cuanto a la detección de resistencia a los fármacos antituberculosos, se cuenta con el método de proporciones múltiples, el cual constituye la prueba de referencia, pero cuyos resultados se tardan de cuatro a seis semanas (9). Otras pruebas comerciales más rápidas, como el BACTEC ${ }^{\mathrm{TM}}$ 460TB y el BACTEC ${ }^{\text {TM }}$ MGIT (10), se basan en el crecimiento bacteriano en presencia del antibiótico, y el tiempo de respuesta oscila entre 11 y 14 días. También están las técnicas basadas en la amplificación de ácidos nucleicos, las cuales se han convertido en una importante herramienta diagnóstica; entre estas pueden mencionarse el test INNOLipa ${ }^{\text {Tм }}$ Rif.TB (Inmunogenetics) (11), el GenoType ${ }^{\mathrm{TM}}$ MTBDRplus y el GenoType Mycobacteria Direct Test $^{\circledR}$ (Hain Lifescience, Nehren, Germany) $(12,13)$.

Todas estas pruebas evalúan la presencia de la micobacteria, pero las dos primeras detectan la resistencia mediante la determinación de mutaciones genéticas y, a pesar de ser tan rápidas como los métodos de crecimiento bacteriano, requieren personal entrenado, infraestructura adecuada, reactivos y equipos sofisticados, y tienen un costo considerable, lo cual dificulta su uso como pruebas de rutina para la mayoría de las instituciones prestadoras de salud del país, con excepción de algunos hospitales privados, que tienen la suficiente capacidad económica, y de laboratorios centrales de carácter público que ya cuentan con algunas plataformas diagnósticas de biología molecular.

La prueba molecular Xpert ${ }^{\circledR}$ MTB/RIF ha significado un avance importante en el diagnóstico de la tuberculosis. La prueba recurre a una plataforma automatizada con múltiples funciones, la cual purifica, concentra e identifica las secuencias de ácidos nucleicos de $M$. tuberculosis mediante una reacción en cadena de la polimerasa en tiempo real (PCR-RT) y, simultáneamente, determina la sensibilidad a rifampicina en aproximadamente dos horas con una muestra directa (14); además, el ensayo provee información semicuantitativa de la carga de micobacterias, la cual constituye un biomarcador importante para determinar la gravedad de la enfermedad y evaluar el riesgo de trasmisión (15).
La prueba Xpert ${ }^{\circledR}$ MTB/RIF fue aprobada por la OMS para la evaluación de muestras pulmonares en diciembre de 2010, especialmente en entornos con altas tasas de tuberculosis asociada al HIV y de tuberculosis multirresistente (16). En varios estudios clínicos de tuberculosis pulmonar se ha encontrado una sensibilidad de 99 a $100 \%$ con baciloscopia positiva y de 57 a $83 \%$ con baciloscopia negativa (17). Además, en investigaciones recientes con esta prueba molecular se ha incluido una variedad de muestras clínicas de sitios diferentes a las vías respiratorias de pacientes con tuberculosis extrapulmonar (18-20). A finales del 2013, la OMS amplió sus recomendaciones sobre el uso de la Xpert ${ }^{\circledR}$ MTB/RIF e incluyó el diagnóstico de la tuberculosis en niños, así como de algunas formas de tuberculosis extrapulmonar (21).

El uso de la prueba Xpert $^{\circledR}$ MTB/RIF en especímenes extrapulmonares es mucho más complejo que en las muestras de pacientes con la enfermedad pulmonar, debido a la diversidad y las características de las muestras clínicas, por ejemplo, las dificultades para obtener los tejidos adecuados para los análisis y los volúmenes mínimos de los líquidos. El principal reto es la necesidad de proporcionar un estándar riguroso previo al análisis en la plataforma, dada su amplia gama de formas de procesamiento.

Por ello, el propósito de este estudio fue evaluar el test $\mathrm{Xpert}^{\circledR} \mathrm{MTB} / \mathrm{RIF}$ en muestras extrapulmonares para la detección de $M$. tuberculosis y de la sensibilidad a rifampicina en pacientes atendidos en un hospital de alta complejidad de Medellín, con el fin de comprobar la utilidad de la prueba como apoyo en el diagnóstico de la tuberculosis, con miras a su implementación rutinaria en el laboratorio de microbiología y biología molecular.

\section{Materiales y métodos}

Se hizo un estudio descriptivo de corte trasversal y recopilación ambispectiva de los datos correspondientes al periodo de febrero de 2014 a octubre de 2013 (hacia atrás) y hasta julio de 2014 (hacia adelante). La población de estudio incluyó a los pacientes con sospecha clínica de tuberculosis extrapulmonar con base en los siguientes criterios de inclusión: las muestras de los pacientes debían tener baciloscopia con tinción de ZiehlNeelsen, inoculación en cultivo Ogawa-Kudoh y prueba molecular Xpert ${ }^{\circledR}$ MTB/RIF, y debían ser extrapulmonares y provenir de adultos o de niños atendidos durante el periodo de estudio en el Hospital Universitario de San Vicente Fundación 
en Medellín. Se excluyeron aquellos aislamientos cuyo cultivo para micobacterias evidenció contaminación en el momento de la lectura, así como los de los pacientes cuya historia clínica no tuviera la información completa.

\section{Información clínica y epidemiológica}

La información clínica y epidemiológica se obtuvo de las historias clínicas electrónicas de los pacientes y, posteriormente, se consignó en el formulario diseñado para el estudio. Los datos obtenidos incluían las variables sociodemográficas, el diagnóstico de base y los resultados positivos en la prueba Xpert $^{\circledR}$ MTB/RIF. En cuanto a los resultados microbiológicos, se revisaron los registros manuales y los del sistema de información del Laboratorio de Micobacterias y Biología Molecular de la institución.

\section{Baciloscopia y cultivo}

Los diferentes tipos de tejidos se maceraron en una suspensión de $2 \mathrm{ml}$ de solución salina, y los líquidos se centrifugaron a 3.500 gravedades durante 30 minutos en una centrífuga refrigerada. La técnica de tinción utilizada fue la tradicional de Ziehl-Neelsen. Todas las muestras fueron descontaminadas con hidróxido de sodio al $4 \%$ (22), a excepción de los líquidos que no presentaban turbidez después de la centrifugación. Posteriormente, las muestras se cultivaron con el método de Kudoh en dos tubos de medio de Ogawa-Kudoh, de acuerdo con el manual de la Organización Panamericana de la Salud (OPS) (22). Los medios se incubaron a $37{ }^{\circ} \mathrm{C}$ en posición horizontal y la lectura se hizo semanalmente; si al cabo de la octava semana no se observaba crecimiento, se reportaban como negativos.

Tratamiento previo de las muestras clínicas para la prueba molecular

En el momento del cultivo y la tinción de ZiehlNeelsen en el laboratorio de micobacterias, una parte de cada muestra se almacenó a una temperatura entre 2 y $8{ }^{\circ} \mathrm{C}$ durante un máximo de 48 horas para su posterior procesamiento con la prueba Xpert ${ }^{\boxplus}$ MTB/RIF.

El procedimiento adaptado para los tejidos consistió en la homogenización mecánica en pequeñas piezas y su posterior maceramiento en $2 \mathrm{ml}$ de solución salina amortiguada por fosfatos (Phosphate Buffered Saline, PBS). Esta suspensión se pasó a un tubo de $15 \mathrm{ml}$ y se le adicionó hidróxido de sodio al $4 \%$ en una proporción de
1 a 1; esta mezcla luego se homogeneizó en un mezclador de vórtice durante 20 segundos y se incubó durante 15 minutos a temperatura ambiente. A continuación, se centri-fugó durante 15 minutos a $3.000 \mathrm{~g}$ y temperatura ambiente, $y$ se dejó reposar por cinco minutos. Se descartó el sobrenadante y el pellet se suspendió de nuevo en $2,5 \mathrm{ml}$ del reactivo del kit. Esta mezcla se homogeneizó en un agitador de vórtice por 5 segundos y se dejó en reposo durante 15 minutos a temperatura ambiente. Posteriormente, $2 \mathrm{ml}$ de esta suspensión se añadieron al cartucho de reacción de la prueba Xpert ${ }^{\circledR}$ MTB/RIF.

Las muestras de los líquidos cefalorraquídeo, pleural, sinovial, peritoneal y pericárdico que presentaban turbidez, se procesaron igual que las de los tejidos, y aquellos que no presentaban turbidez, se concentraron por centrifugación y se adicionaron directamente al cartucho. Los que presentaban un volumen inferior a $2 \mathrm{ml}$ se completaron con los reactivos hasta alcanzar $2,5 \mathrm{ml}$.

\section{GeneXpert Dx System, versión 4.3}

Una vez se colocaron los cartuchos en el instrumento, se inició el protocolo de corrido y análisis automático. La prueba tiene como blanco el gen rрoB de la micobacteria. Los resultados de $M$. tuberculosis se registraron como "detectado" o "no detectado" en uno de cuatro niveles: muy bajo, bajo, medio o alto, en tanto que la rifampicina se reportó como sensible o resistente. Un control de extracción y amplificación viene integrado en el cartucho, lo que garantiza la validez de los resultados.

\section{Sensibilidad de los fármacos antituberculosos}

Los cultivos con medio de Ogawa-Kudoh en los que hubo crecimiento de $M$. tuberculosis se enviaron al Laboratorio Departamental de Salud Pública, en donde se utilizó el método de proporciones múltiples (9) para detectar la sensibilidad a los medicamentos antituberculosos de primera línea, entre ellos, la rifampicina.

\section{Análisis de la información}

Los datos se sistematizaron con el programa Statistical Package for the Social Sciences ${ }^{\circledR}$ para Windows, versión 20.0, mediante el cual se calcularon las medidas de frecuencia, la tendencia central y la dispersión, y se elaboraron cuadros para la caracterización de la población de estudio. El programa Epidat 3.1 de la OPS se utilizó para los cálculos de validez (sensibilidad, especificidad 
y valores predictivos), así como del índice kappa de concordancia, y de sus respectivos intervalos de confianza. El cultivo en medio de Ogawa-Kudoh se empleó como método de referencia para el cálculo de la sensibilidad, la especificidad y los valores predictivos de la prueba molecular.

\section{Consideraciones éticas e institucionales}

De acuerdo con la Resolución 008430 de 1993 del Ministerio de Salud de Colombia, el presente estudio se clasificó como una investigación sin riesgo, ya que la información requerida provenía de una fuente secundaria. Se contó con la aprobación de la Unidad de Investigaciones y del Comité de Ética de la institución, y con el aval de la Dirección del Hospital, para adelantar el estudio y redactar el manuscrito.

\section{Resultados}

Se incluyeron 376 muestras en el estudio, de las cuales cuatro se excluyeron debido a la contaminación del cultivo, por lo que se evaluaron finalmente 372 muestras de 301 pacientes, pues a algunos de ellos se les tomaron dos o más para los análisis debido a la sospecha de la presencia de tuberculosis durante el periodo de estudio. Del total de muestras, 182 (60\%) provenían de hombres; el promedio de edad en ambos sexos fue de 42 años $( \pm 19,35)$, y en $50 \%$ de los pacientes fue de hasta 41,5 años (edad mínima: un año, edad máxima: 91 años). La edad más frecuente en los hombres fue 39 años, mientras que en las mujeres fue de 36 años; el diagnóstico más frecuente fue el de la infección por HIV, con 121 casos (40 \%) (cuadro 1).

Los resultados de los análisis se presentan para las muestras y los pacientes. De los 372 especímenes analizados, $39(10,5 \%)$ fueron positivos para $M$. tuberculosis en la prueba Xpert ${ }^{\circledR}$ MTB/RIF, y 23 de las muestras, sobre todo de tejidos, provenían de pacientes con diagnóstico de infección por HIV, con 27 detecciones (7,2\%), seguidas de líquido cefalorraquídeo con siete $(1,8 \%)$. El cultivo fue positivo en 31 muestras (8,3\%); 22 de estos aislamientos correspondían a tejidos totales $(6 \%)$ y nueve (3\%) eran de líquidos; 12 muestras tuvieron baciloscopia positiva (3,2\%) (figura 1). La prueba molecular de dos de las muestras fue negativa, pero el cultivo fue positivo (cuadro 2).

La sensibilidad y la especificidad de la prueba Xpert $^{\circledR}$ MTB/RIF comparadas con las del cultivo fueron de $93,5 \%\left(\mathrm{IC}_{95 \%} 83,2-100\right)$ y de $97 \%$ $\left(\mathrm{IC}_{95 \%}\right.$ 95,1-99), respectivamente; su valor predictivo positivo fue de $74,3 \%\left(\mathrm{IC}_{95 \%} 59,3-89,3,\right)$
Cuadro 1. Características demográficas y clínicas de los pacientes con sospecha de tuberculosis extrapulmonar durante el periodo de estudio

\begin{tabular}{lcc}
\hline Características & \multicolumn{2}{c}{ Total (n=301) } \\
\cline { 2 - 3 } & $\mathbf{n}$ & $\%$ \\
\hline Sexo & 182 & \\
$\quad$ Hombres & 119 & 60,5 \\
$\quad$ Mujeres & & 39,5 \\
Edad (años) & 22 & \\
0 a 14 & 32 & 7,3 \\
15 a 24 & 62 & 10,6 \\
25 a 34 & 59 & 20,5 \\
35 a 44 & 49 & 19,6 \\
45 a 55 & 77 & 16,2 \\
56 y más & & 25,5 \\
Diagnóstico base & 121 & 40,1 \\
Infección por HIV & 12 & 4,0 \\
Diabetes & & \\
\hline
\end{tabular}

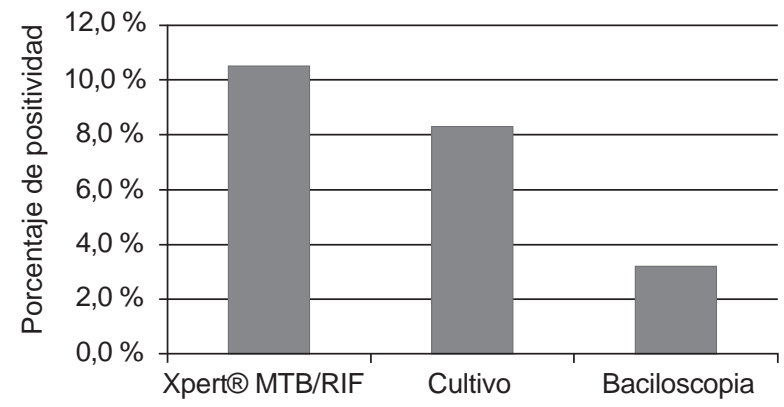

Figura 1. Porcentaje de positividad de Mycobacterium tuberculosis en la prueba molecular y en las pruebas convencionales

$y$ el negativo, de 99,4\% (IC $\left.{ }_{95 \%} 98,4-100\right)$. El análisis estratificado por sitio anatómico evidenció sensibilidades mayores de $75 \%$ para los líquidos y para los tejidos, pero no se pudo determinar en abscesos debido a las pocas muestras. La especificidad estratificada para ocho de los 12 tipos diferentes de muestras, se registró en un rango entre 66 y $100 \%$ (cuadro 2).

La prueba Xpert ${ }^{\circledR}$ MTB/RIF tuvo una sensibilidad de $100 \%$ ( $\left.\mathrm{IC}_{95 \%} 95-100\right)$, y una especificidad de $92 \%\left(\mathrm{IC}_{95 \%}\right.$ 89-95) comparada con los resultados de la baciloscopia, en tanto que la sensibilidad de la baciloscopia con respecto al cultivo fue de $38,7 \%\left(\mathrm{IC}_{95 \%}\right.$ 19,5-57,4), con una especificidad de $100 \%\left(\mathrm{IC}_{95 \%}\right.$ 99,8-100); los índices kappa en las pruebas evaluadas, se muestran en el cuadro 3.

En cuanto a los resultados concernientes a la rifampicina, con la prueba Xpert ${ }^{\circledR}$ MTB/RIF se determinó que 37 muestras eran sensibles, y en una no se pudo determinar el resultado, pero el cultivo fue negativo; mediante la comparación 
Cuadro 2. Resultados de la prueba Xpert® MTB/RIF según el cultivo y el tipo de muestra

\begin{tabular}{|c|c|c|c|c|c|c|c|}
\hline \multirow[t]{2}{*}{ Muestra clínica } & \multicolumn{2}{|c|}{ Cultivo positivo } & \multicolumn{2}{|c|}{ Cultivo negativo } & \multirow[t]{2}{*}{ Total } & \multirow{2}{*}{$\begin{array}{l}\text { Sensibilidad } \\
\quad \%\left(\text { IC }_{95 \%}\right)\end{array}$} & \multirow{2}{*}{$\begin{array}{l}\text { Especificidad } \\
\quad \%\left(\text { IC }_{95 \%}\right)\end{array}$} \\
\hline & $\begin{array}{c}\text { Xpert } \\
\text { positivo }\end{array}$ & $\begin{array}{c}\text { Xpert } \\
\text { negativo }\end{array}$ & $\begin{array}{c}\text { Xpert } \\
\text { positivo }\end{array}$ & $\begin{array}{c}\text { Xpert } \\
\text { negativo }\end{array}$ & & & \\
\hline \multicolumn{8}{|l|}{ Líquidos estériles } \\
\hline Líquido cefalorraquídeo & 6 & 0 & 1 & 148 & 155 & $100(91-100)$ & $99(97-100)$ \\
\hline Líquido pleural & 2 & 0 & 0 & 46 & 48 & $100(75-100)$ & $100(98-100)$ \\
\hline Líquido sinovial & 0 & 0 & 1 & 3 & 4 & $100(50-100)$ & $100(87-100)$ \\
\hline Líquido peritoneal & 1 & 0 & 0 & 14 & 15 & $100(50-100)$ & $100(96-100)$ \\
\hline Líquido pericárdico & 0 & 0 & 0 & 2 & 2 & * & * \\
\hline \multicolumn{8}{|l|}{ Tejidos } \\
\hline Ganglio & 3 & 1 & 1 & 2 & 7 & $75(20-100)$ & $66(0-100)$ \\
\hline Pulmón & 3 & 0 & 0 & 1 & 4 & $100(83-100)$ & $100(50-100)$ \\
\hline Hueso & 1 & 0 & 0 & 4 & 5 & $100(50-100)$ & $100(87-100)$ \\
\hline No especificados & 12 & 0 & 6 & 108 & 126 & $100(95-100)$ & $94(90-99)$ \\
\hline \multicolumn{8}{|l|}{ Otros } \\
\hline Abscesos & 0 & 1 & 1 & 0 & 2 & * & * \\
\hline Jugo gástrico & 0 & 0 & 0 & 3 & 3 & * & * \\
\hline Tejido gastrointestinal & 1 & 0 & 0 & 0 & 1 & * & * \\
\hline Total & 29 & 2 & 10 & 331 & 372 & $94(83-100)$ & 97 (95-99) \\
\hline
\end{tabular}

*Sensibilidad y especificidad no calculables

Cuadro 3. Resultados del índice kappa

\begin{tabular}{lc}
\hline Métodos diagnósticos & Índice kappa $\left(\right.$ IC $\left._{95 \%}\right)$ \\
\hline Baciloscopia Vs. cultivo & $0,5366(0,3567-0,7165)$ \\
Xpert $^{\boxplus}$ MTB/RIF Vs. baciloscopia & $0,4431(0,2751-0,6111)$ \\
Xpert $^{\boxplus}$ MTB/RIF Vs. cultivo & $0,811 \quad(0,7074-0,9147)$ \\
\hline
\end{tabular}

con el método de proporciones, se encontró una concordancia de $100 \%$ en la sensibilidad a la rifampicina en los 29 aislamientos positivos por cultivo y prueba molecular. Una muestra de líquido cefalorraquídeo resultó resistente a rifampicina en la prueba de Xpert $^{\circledR}$ MTB/RIF y mediante análisis fenotípico se comprobó también su resistencia a isoniacida por el método de proporciones y por el método automatizado BD BACTECTM MGIT 960 aplicado en el Instituto Nacional de Salud. Ningún aislamiento presentó resistencia solo a isoniacida mediante el método fenotípico.

Con la prueba $\mathrm{Xpert}^{\circledR}$ MTB/RIF se obtuvieron, asimismo, resultados semicuantitativos en las muestras positivas con base en la cantidad de material genético de la micobacteria: en 27 detecciones (70\%), los resultados fueron de "bajo" y "muy bajo", se correspondieron con los resultados negativos en la baciloscopia.

En cuanto al tiempo de obtención de resultados, la determinación de la presencia o ausencia de la micobacteria y su sensibilidad a la rifampicina en los casos positivos tardó, en promedio, dos días $( \pm 1,2)$; en cuanto al cultivo, los resultados se obtuvieron en un promedio de 27 días $( \pm 6,2)$ para los aislamientos positivos, y se necesitaron hasta 60 días $( \pm 12)$ para determinar la sensibilidad a los medicamentos de primera línea utilizando el método de proporciones. Con respecto al impacto de los resultados positivos en el manejo clínico de los 34 pacientes (39 detecciones), se encontró que el resultado de la prueba Xpert ${ }^{\circledR}$ MTB/RIF en siete de ellos $(20,5 \%)$ fue definitivo para establecer el diagnóstico o influyó en las decisiones sobre el tratamiento, mientras que en el resto de casos sirvió para confirmar la sospecha clínica.

\section{Discusión}

El diagnóstico precoz y el tratamiento inicial de la tuberculosis son estrategias críticas de suma importancia en el manejo de este problema de salud pública. Además de las pruebas tradicionales, los Centers for Disease Control and Prevention (CDC) recomiendan la detección de ácidos nucleicos desde el año 2000 (23).

En este artículo se presentan los resultados de la primera aplicación de la plataforma Xpert ${ }^{\circledR}$ MTB/ RIF en especímenes no respiratorios tomados de diferentes sitios anatómicos de pacientes pediátricos y adultos en el contexto de un hospital de alta complejidad, y de su comparación con los métodos microbiológicos convencionales para el diagnóstico de la tuberculosis extrapulmonar. Como se sabe, el diagnóstico de esta forma de tuberculosis es difícil, ya que el número de bacterias presentes es muy bajo $y$, además, se requieren 
procedimientos invasivos para tomar las muestras y no es fácil obtenerlas en número suficiente, lo que convierte a la plataforma $\mathrm{Xpert}^{\circledR} \mathrm{MTB} / \mathrm{RIF}$ en una herramienta muy útil (24).

La mayoría de los pacientes del estudio presentaba un diagnóstico de base de infección por HIV, y $58,9 \%$ de ellos tuvo resultados positivos en la prueba Xpert ${ }^{\circledR}$ MTB/RIF (23 muestras de 22 pacientes). Esta distribución coincidió con lo que se ha informado para este tipo de población: de acuerdo con la OMS, en el 2014 se presentó un aumento de $43,9 \%$ en los casos de tuberculosis asociada con la infección por HIV con respecto al año anterior (2), asociación que es la más frecuente en el caso de la tuberculosis extrapulmonar, como se ha evidenciado en varios estudios locales en instituciones de alta complejidad, en los cuales se registraron diferencias significativas con respecto a los pacientes inmunocompetentes (25). Por ello, el médico siempre debe sospechar su presencia; se sabe que en los pacientes con infección por HIV se debe buscar tuberculosis, y viceversa (26).

En otros trabajos se ha reportado como una de las bondades de esta plataforma de diagnóstico su mayor sensibilidad frente a la baciloscopia; además, su valor predictivo es mayor, sobre todo en pacientes con infección por HIV, con la consecuente reducción de la tasas de mortalidad y morbilidad $(27,28)$. En el caso del presente estudio, se logró un diagnóstico rápido de tuberculosis extrapulmonar con muestras de diferentes tejidos y líquidos, de las cuales diez tuvieron baciloscopia negativa y cultivo positivo, y una de ellas, de líquido cefalorraquídeo, mostró resistencia a la rifampicina.

Por otro lado, la diabetes, que fue el segundo diagnóstico inicial más frecuente en los pacientes incluidos, generalmente se reporta como un factor predisponente para el desarrollo de tuberculosis pulmonar (29), pero también, como un factor de riesgo para el desarrollo de tuberculosis miliar, conjuntamente con otras variables como la malnutrición, el alcoholismo, la falla renal crónica, los trasplantes y el uso de medicamentos inmunosupresores (30). En este estudio, solo un paciente con este diagnóstico de base dio positivo en la prueba molecular en una muestra de tejido no especificado.

En cuanto al aporte del estudio en las muestras tomadas en niños, si bien solo se registraron dos casos positivos mediante la prueba Xpert $^{\circledR}$ MTB/ RIF (en líquido cefalorraquídeo y en tejido no especificado), este fue significativo por su utilidad dada la naturaleza paucibacilar de la mayoría de los casos de tuberculosis infantil y de la forma extrapulmonar. El desempeño de las pruebas moleculares para la detección de tuberculosis en niños no se ha evaluado a fondo, aunque en algunos estudios anteriores se sugiere que este sería similar al de la prueba con la baciloscopia negativa en adultos (23). En el caso específico de la prueba Xpert ${ }^{\circledR}$ MTB/RIF, en un estudio del 2012 se demostró una sensibilidad de 86,9\% $\left(\mathrm{IC}_{95 \%} 80-93\right)$ en muestras no respiratorias (20), lo que significó un avance importante en el ámbito del diagnóstico pediátrico, que sigue siendo un gran desafío. Por otro lado, la OMS recomienda la prueba Xpert $^{\circledR}$ MTB/RIF para el diagnóstico de niños con sospecha de tuberculosis pulmonar y extrapulmonar (21) .

La prueba Xpert $^{\circledR}$ MTB/RIF fue aprobada por la OMS para el diagnóstico de tuberculosis en países con prevalencia baja y media (18). En lo que se refiere a su sensibilidad y especificidad en muestras no respiratorias, se han encontrado registros de sensibilidad de $59 \%$ y de especificidad de $92 \%$ en países con mucha prevalencia (19). En el 2013 se notificaron en Colombia 12.062 casos de tuberculosis en todas sus formas, lo que corresponde a una incidencia intermedia; de dichos casos, $80,8 \%$ fue de tuberculosis pulmonar, $14,6 \%$ de tuberculosis extrapulmonar y $4,5 \%$ de tuberculosis meníngea (2). En Medellín, el informe epidemiológico de 2014 daba cuenta de un número de casos que ubicó a la ciudad como un territorio de alto riesgo de transmisión, pues superaba los 50 casos por 100.000 habitantes $(63,8)$, con una tendencia a la estabilización (31).

Por otro lado, en contextos de baja prevalencia, Tortoli, et al., evaluaron la utilidad de la prueba en muestras diferentes a esputo, para un total de 1.476 muestras extrapulmonares de diversos sitios anatómicos, y obtuvieron una sensibilidad global de $81,3 \%\left(\mathrm{IC}_{95 \%} 76,2-85,8\right)$ y una especificidad de 99,8\%, (IC ${ }_{95 \%} 99,4-100$ ) (20); su estudio, al igual que este, incluyó a niños. La sensibilidad general del presente estudio, sin embargo, superó ligeramente la obtenida por Tortoli, et al., con $94 \%$ $\left(\mathrm{IC}_{95 \%}\right.$ 83-100), en tanto que la especificidad fue de $97 \%\left(\mathrm{IC}_{95 \%}\right.$ 95-99). De igual manera, en un estudio llevado a cabo en España, Clemente, et al., encontraron una sensibilidad general de 73,6 \% y una especificidad de 99,9\% (32). En otro reporte también se obtuvieron resultados concordantes con este, con una sensibilidad de $95 \%\left(\mathrm{IC}_{95 \%} 87\right.$ 100) y una especificidad de $100 \%\left(\mathrm{IC}_{95 \%} 99-100\right)$, 
pero dicho estudio no incluyó los análisis por sitio anatómico (33). En un estudio anterior de ámbito local, con 144 muestras extrapulmonares, la sensibilidad general fue de $70 \%$ y la especificidad fue de $97,7 \%$, y aunque en él no se especificó el origen de las muestras (34), es comparable con el presente trabajo en el sentido de que la mínima sensibilidad obtenida fue de $75 \%$.

Con respecto al porcentaje de positividad, se encontró una mayor frecuencia en las formas tisulares, en tejidos no especificados o en los provenientes de ganglio. Es de resaltar que en los líquidos evaluados en este trabajo, con excepción del pericárdico, las sensibilidades fueron de $100 \%$, es decir, que superaron las de otros estudios, las cuales han oscilado entre 40,5 y $59 \%$ en este tipo de muestras $(19,35)$. Sin embargo, en los líquidos pleural, sinovial, peritoneal y pericárdico, los resultados no fueron concluyentes debido al número limitado de muestras analizadas durante el periodo de estudio; no obstante, hubo una buena concordancia con el método de referencia. Los datos locales han evidenciado una tasa de 14,5\% de tuberculosis extrapulmonar (2).

En los artículos consultados, la tuberculosis pleural se describe como una de las formas de localización extrapulmonar más frecuentes en el mundo, seguida por la forma linfática, y ambas constituyen las variantes de mayor predominio en pacientes jóvenes (8). La primera, además, es la causa más frecuente de derrame pleural en lugares de mucha prevalencia de infección por HIV (36). Según tres reportes previos, no se han registrado beneficios importantes al procesar con $\mathrm{Xpert}^{\circledR} \mathrm{MTB} / \mathrm{RIF}$ los líquidos pleurales, pues en ellos los valores de sensibilidad fueron de $15 \%$ (37), $25 \%$ (38) y $33 \%$ (20), en contraste con lo obtenido en este estudio, en el que la sensibilidad fue de $100 \%\left(\mathrm{IC}_{95 \%} 75\right.$ $100)$, al igual que la especificidad ( $\mathrm{IC}_{95 \%}$ 98-100). Aunque se procesaron 48 muestras de este tipo, y a pesar de ser el segundo líquido más frecuente entre las muestras analizadas, dichos resultados no son concluyentes en términos de rendimiento; por otro lado, el tratamiento previo descrito en la metodología permitió procesar volúmenes de más de $3 \mathrm{ml}$.

En lo que concierne a la presentación meníngea, se ha reportado que esta representa, aproximadamente, $1 \%$ de la prevalencia de tuberculosis, la cual es una de las más devastadoras por su elevada mortalidad (20 a $50 \%$ ) y por las graves secuelas neurológicas en quienes sobreviven (39); además, debido a la poca sensibilidad de la baciloscopia, que puede llegar a ser de tan solo $20 \%$, su pronóstico empeora por la tardanza en el diagnóstico y, por ende, en el inicio del tratamiento (40). Según los informes del 2013, en Colombia esta forma de la enfermedad representó $4,5 \%$ de su prevalencia (2); en este sentido, el desempeño de la prueba Xpert ${ }^{\circledR}$ MTB/RIF en este estudio fue contundente, pues se obtuvo un resultado más rápido para las 155 muestras de líquido cefalorraquídeo y se detectó la resistencia en un caso, proceso que habría tardado más de 30 días por los métodos convencionales de aislamiento y reporte farmacológico de la bacteria. Asimismo, la prueba tuvo una muy buena sensibilidad, de $100 \%\left(\mathrm{IC}_{95 \%} 91-100\right)$, en tanto que en el estudio de Denkinger, et al., fue de $81 \%\left(\mathrm{IC}_{95 \%}\right.$ 59-92) (41). En este tipo de líquidos es crucial el volumen disponible para el procesamiento, el cual, en nuestro estudio, fluctuó entre $500 \mu \mathrm{l}$ y $1 \mathrm{ml}$, y se adicionó directamente al cartucho; sin embargo, Denkinger, et al., reportaron un incremento de la sensibilidad cuando usaron la centrífuga, lo cual no se hizo en el presente estudio en los líquidos con volúmenes inferiores a $2 \mathrm{ml}$.

En lo que se refiere a las muestras de tejidos, si bien en un gran número de estas no se especificaba de qué tejidos provenían, se obtuvo una buena sensibilidad (entre 75 y $100 \%$ ). En otro estudio en el cual no se precisaron los sitios anatómicos de donde provenían las muestras, aunque se supone que incluían los ganglios, la estimación conjunta estuvo por encima de $88 \%$ ( $\left.\mathrm{IC}_{95 \%} 82-95\right)$ (20).

Estos hallazgos en las muestras de líquido cefalorraquídeo y de tejidos, concuerdan con las recomendaciones de la OMS para el uso de la prueba Xpert ${ }^{\circledast}$ MTB/RIF en el diagnóstico de tuberculosis meníngea y las formas tisulares (21), en particular en la primera, debido a que en los pacientes con sospecha de meningitis tuberculosa se requiere un diagnóstico rápido.

A pesar del escaso número de casos positivos (10,5\% del total de muestras evaluadas), el tiempo y la oportunidad de respuesta tanto para resultados positivos como negativos disminuyeron considerablemente, y la prueba molecular superó casi tres veces los resultados positivos obtenidos mediante la baciloscopia; además, debe considerarse que en la tuberculosis extrapulmonar el desempeño de las pruebas de microscopía depende del tipo de muestra, pues es mejor en aquellas obtenidas por biopsia de tejidos $(>70-80 \%)$, y es manifiestamente menor en líquidos biológicos (5-20\%) (42). Por otra parte, 
la baciloscopia negativa no debe considerarse como un diagnóstico definitivo. Otras ventajas de la prueba son que sirve para medir la sensibilidad al medicamento de primera línea, la rifampicina, y que, en los aislamientos con resultados positivos en el cultivo y por el método de proporciones, también predijo la sensibilidad a la isoniacida en $100 \%$ de las cepas evaluadas.

En cuanto a la información cuantitativa de la prueba Xpert ${ }^{\circledR}$ MTB/RIF, en 27 detecciones (70\%) el resultado fue "bajo" y "muy bajo", y correspondía a las baciloscopias negativas. Este hallazgo es importante porque se ajustó a lo esperado dada la naturaleza paucibacilar de la tuberculosis extrapulmonar, y porque también evalúo de manera indirecta la calidad de la baciloscopia en términos de su lectura y de la técnica empleadas en el proceso. En un estudio previo, específicamente en muestras respiratorias, esta información se planteó como otra variable que debe incorporarse en los informes de salud pública por su fuerte correlación con las técnicas de microscopía (15).

Con respecto a los falsos negativos (dos casos en este estudio), otros autores han sugerido que en las pruebas moleculares tales resultados se deben a la presencia de inhibidores o a errores técnicos de laboratorio (43). También, se ha documentado que la descontaminación de muestras estériles y no estériles puede disminuir la carga bacilar y, por consiguiente, reducir la sensibilidad del test (24). En este estudio, el tamaño y el volumen pudieron haber sido un aspecto crítico, ya que según el protocolo del laboratorio de micobacteriología, deben garantizarse al máximo para las pruebas microbiológicas convencionales, en particular, cuando las muestras son de difícil obtención. En la dinámica rutinaria de trabajo, se deja una muy pequeña proporción para la prueba molecular, lo cual genera resultados indeterminados para la interpretación de la sensibilidad a la rifampicina en la plataforma diagnóstica, lo que se evidenció solo en una muestra en este trabajo. El resultado clínico de uno de los casos se logró con base en los hallazgos histológicos, radiológicos y epidemiológicos durante la hospitalización, en tanto que los hallazgos microbiológicos no se tuvieron en cuenta para decidir el tratamiento antituberculoso. En el otro caso, el paciente se atendió en consulta ambulatoria, pues su estado de salud no presentaba un deterioro acusado, pero su diagnóstico no se determinó hasta obtener los resultados de las biopsias.
Por otro lado, los resultados positivos obtenidos mediante la prueba Xpert ${ }^{\circledast}$ MTB/RIF en los cultivos negativos (correspondientes a diez muestras en este estudio), se deben a una de las limitaciones de las pruebas basadas en la detección de ácidos nucleicos, pues no diferencian los bacilos viables de los no viables, situación que ya se ha reportado en pacientes bajo tratamiento farmacológico en quienes se usa la prueba (44), ya que podría llevar a que los resultados se interpretaran como falsos positivos; sin embargo, el sistema de extracción, amplificación e interpretación de resultados es cerrado, lo cual minimiza considerablemente la contaminación cruzada. Estas situaciones resaltan el hecho de que las pruebas moleculares no reemplazan los métodos convencionales de diagnóstico micobacteriológico, y que pueden ser mitigadas cuando se combinan con los hallazgos epidemiológicos, clínicos y de laboratorio del paciente. Según las recomendaciones de los CDC, en aquellos casos cuya baciloscopia sea negativa con un nivel elevado o medio de sospecha clínica de tuberculosis y resultados positivos en la prueba molecular, el diagnóstico debe considerarse como presuntivo de tuberculosis (45); en este sentido, el resultado clínico de los diez casos, todos con baciloscopia negativa y factores epidemiológicos asociados a tuberculosis extrapulmonar, permitió confirmar el diagnóstico.

Las 331 pruebas negativas representan un porcentaje alto y, según la revisión de trabajos previos, las implicaciones clínicas de un resultado negativo no son sencillas de interpretar. La utilidad de un resultado negativo para descartar la tuberculosis extrapulmonar depende del sitio anatómico del que provenga la muestra, de las características clínicas del paciente (por ejemplo su estado inmunológico) y de la prevalencia subyacente de la tuberculosis en la población, entre otros aspectos (46). En este sentido, en todos los pacientes incluidos en el presente estudio se sospechaba la presencia de tuberculosis extrapulmonar, pero, dado que estas pruebas no reemplazan los métodos convencionales, los pacientes se manejaron según el riesgo de tener la enfermedad y los diagnósticos diferenciales. En cuanto al impacto de los resultados negativos, el presente trabajo no lo consideró. Según las recomendaciones de la OMS, los individuos con sospecha de tuberculosis extrapulmonar y resultado negativo en la prueba Xpert ${ }^{\circledR}$ MTB/RIF, deben someterse a pruebas diagnósticas adicionales; en cuanto a los pacientes en quienes la sospecha clínica de tener 
la enfermedad es muy grande, especialmente los niños, esta debe tratarse, incluso si el resultado en la Xpert ${ }^{\circledR}$ MTB/RIF es negativo, o si la prueba no está disponible (47).

La mejor concordancia entre las tres herramientas diagnósticas (baciloscopia, cultivo en OgawaKudoh y prueba molecular), se registró entre la prueba Xpert ${ }^{\circledR}$ MTB/RIF y el cultivo (índice kappa $\left.=0,811 ; \quad I C_{95 \%}, 0,7074-0,9147\right)$; hay que tener en cuenta que este último es el método de referencia para el diagnóstico microbiológico y que tiene las limitaciones de tiempo ya mencionadas. En un estudio anterior con otra prueba en tiempo real, se reportó una concordancia intermedia de 0,59 (48) con otro medio sólido (medio de Löwenstein-Jensen), en una comparación similar a la de este trabajo.

La información de los análisis de laboratorio en el presente estudio debe ser insumo para fortalecer las actividades de diagnóstico de las instituciones del país, ya que en 2013 el 75,0 \% de los 12.824 casos de pacientes reportados al Instituto Nacional de Salud se confirmó en el laboratorio, 23,1\% correspondió a casos confirmados por clínica y $1,6 \%$ se confirmó por nexo epidemiológico. De ahí la importancia de documentar la experiencia con los nuevos métodos diagnósticos.

Entre las limitaciones del estudio cabe mencionar tres: en primer lugar, los tejidos no especificados, los cuales no pudieron clasificarse correctamente por inconvenientes del sistema de información y representaban 33,8 \% (126) del total; en segundo lugar, a pesar de que con los métodos fenotípicos se encontró una concordancia de $100 \%$ en las cepas sensibles, la evaluación concluyente del rendimiento de la prueba Xpert $^{\circledR}$ MTB/RIF en aislamientos resistentes a la rifampicina requiere un número de muestras mayor debido a la baja prevalencia de la resistencia evidenciada durante el periodo de estudio: solo una de las muestras fue resistente a rifampicina con confirmación fenotípica. Por último, el método de referencia para los análisis no fue el óptimo, ya que por su naturaleza paucibacilar, en aquellas muestras de origen extrapulmonar se requeriría haber contado, al menos, con medios líquidos de cultivo como el que se emplea en el Mycobacterium Growth Indicador Tube (MGIT), y con la tinción de auramina-rodamina para las baciloscopias. Es importante tener esto en cuenta, pues estas metodologías tienen efectos directos sobre la sensibilidad y la especificidad; sin embargo, en el presente estudio se evidenciaron las bondades de la prueba molecular en términos de tiempo de respuesta y porcentaje de positividad en muestras no pulmonares, además del aporte teórico en términos de la validación clínica en el marco de los métodos tradicionales, como los de la tinción de Ziehl-Neelsen y el medio de cultivo OgawaKudoh, que son de uso rutinario en muchos laboratorios del país.

Por último, además de su interés en salud pública, la tuberculosis es una enfermedad cuya atención está incluida en el Plan Obligatorio de Salud, y corresponde a las instituciones prestadoras de servicios de salud garantizar el diagnóstico y el seguimiento de los casos.

Este estudio representa un aporte teórico y práctico a la evaluación de una prueba molecular ya disponible en el país, la cual se emplea para la detección oportuna de la enfermedad, y contribuye al Plan Estratégico "Colombia libre de tuberculosis, 2010-2015", con el cual se concreta la estrategia de "Alto a la tuberculosis" de la OMS (49).

Como la mayoría de las pruebas moleculares, el método $X$ pert $^{\circledR}$ MTB/RIF en muestras extrapulmonares no reemplaza los métodos convencionales del diagnóstico micobacteriológico, pero sí los complementa y los potencia, aunque es cierto que también tiene limitaciones, que, sin embargo, pueden mitigarse si se combina con los hallazgos epidemiológicos, clínicos y de laboratorio del paciente.

La utilidad de la prueba Xpert ${ }^{\circledR}$ MTB/RIF en el diagnóstico rápido de la tuberculosis extrapulmonar quedó demostrada por ser una alternativa rápida, especialmente en líquido cefalorraquídeo y en tejidos, pero no debe emplearse para descartar el diagnóstico debido a la heterogeneidad inherente de las formas extrapulmonares en aspectos como la obtención, el tamaño y los volúmenes de las muestras. Por otro lado, este estudio aportó un protocolo sencillo para el adecuado tratamiento de las muestras antes del análisis en instituciones públicas y privadas que cuenten con la plataforma diagnóstica Xpert ${ }^{\circledR}$.

Además de la correcta identificación de la bacteria causante de la enfermedad, la prueba permitió determinar la sensibilidad del microorganismo a uno de los antibióticos más importantes en el tratamiento farmacológico, la rifampicina, lo que permite orientar desde el inicio el tratamiento $y$, aunque el número de casos positivos fue bajo, 
se demostró su efecto en el manejo clínico de los pacientes atendidos en una institución de tercer nivel de complejidad.

\section{Agradecimientos}

A las unidades de Microbiología y de Investigaciones del Hospital Universitario de San Vicente Fundación, por el soporte logístico y administrativo.

\section{Conflicto de intereses}

Los autores declaran que no existen conflictos de intereses de ninguna índole.

\section{Financiación}

Este estudio fue financiado con recursos del Hospital Universitario de San Vicente Fundación.

\section{Referencias}

1. World Health Organization. Twentieth global report on tuberculosis. Report number WHO/HTM/TB/2015.22. Geneva: World Health Organization; 2015. Fecha de consulta: 7 de diciembre de 2015. Disponible en: http://apps.who.int/iris/bit stream/10665/191102/1/9789241565059_eng.pdf.

2. Instituto Nacional de Salud. Informe final tuberculosis, Colombia, 2014. Fecha de consulta: 1 de diciembre de 2015. Disponible en: http://www.ins.gov.co/lineas-de-accion/ Subdireccion-Vigilancia/Informe\%20de\%20Evento\%20 Epidemiolgico/Tuberculosis\%202014.pdf.

3. Lado-Lado FL, Prieto-Martínez A, Cabarcos-Ortiz De Barrón A, Carballo-Arceo E, Barrio-Gómez E. Recurrence of tuberculosis in patients infected with the human immunodeficiency virus. An Med Interna. 2001;18:243-7.

4. Golden MP, Vikram HR. Extrapulmonary tuberculosis: An overview. Am Fam Physician. 2005;72:1761-8.

5. Raviglione MC, O'Brien R. Mycobacterial diseases. In: Harrison's Principles of Internal Medicine. 18th edition. New York: McGraw-Hill; 2012. p. 1340-59.

6. Hesselink DA, Yoo SM, Verhoeven GT, Brouwers JW, Smit FJ, van Saase JL. A high prevalence of culturepositive extrapulmonary tuberculosis in a large Dutch teaching hospital. Neth J Med. 2003;61:65-70.

7. Dye C, Scheele S, Dolin P, Pathania V, Raviglione MC. Consensus statement. Global burden of tuberculosis: Estimated incidence, prevalence, and mortality by country. WHO Global Surveillance and Monitoring Project. JAMA 1999;282:677-86. http://dx.doi.org/10.1001/jama.282.7.677

8. Pacheco M, Awad C, Arias G, Ojeda P, Garay M, Lara A, et al. Extrapulmonary tuberculosis. A perspective from a third-level hospital. Rev Colomb Neumol. 2013;25:16-26.

9. Canetti G, Rist N, Grosset J. Measurement of sensitivity of the tuberculous bacillus to antibacillary drugs by the method of proportions. Methodology, resistance criteria, results and interpretation. Rev Tuberc Pneumol (Paris). 1963;27:217-72.

10. Mishra B, Rockey SM, Gupta S, Srinivasa H, Muralidharan S. Multi-drug-resistant tuberculosis: The experience of an urban tertiary care hospital in South India using automated BACTEC 460 TB. Trop Doct. 2012;42:35-7. http://dx.doi org/10.1258/td.2011.110247
11. Skenders GK, Holtz TH, Riekstina V, Leimane V. Implementation of the INNO-LiPA Rif. TB® line-probe assay in rapid detection of multidrug-resistant tuberculosis in Latvia. Int J Tuberc Lung Dis. 2011;15:1546-52. http:// dx.doi.org/10.5588/ijtld.11.0067

12. Barnard M, Gey van Pittius NC, van Helden PD, Bosman M, Coetzee G, Warren RM. The diagnostic performance of the GenoType MTBDRplus version 2 line probe assay is equivalent to that of the Xpert MTB/RIF assay. J Clin Microbiol. 2012;50:3712-6. http://dx.doi.org/10.1128/JCM.01958-12

13. Bicmen C, Gunduz AT, Coskun M, Senol G, Cirak AK, Ozsoz A. Molecular detection and identification of Mycobacterium tuberculosis complex and four clinically important nontuberculous mycobacterial species in smearnegative clinical samples by the genotype mycobacteria direct test. J Clin Microbiol. 2011;4:2874-8. http://dx.doi. org/10.1128/JCM.00612-11

14. Lawn SD, Nicol MP. Xpert® MTB/RIF assay: Development, evaluation and implementation of a new rapid molecular diagnostic for tuberculosis and rifampicin resistance. Future Microbiol. 2011;6:1067-82. http://dx.doi.org/10.2217/ fmb.11.84

15. Blakemore R, Nabeta P, Davidow AL, Vadwai V, Tahirli R, Munsamy V, et al. A multisite assessment of the quantitative capabilities of the Xpert MTB/RIF assay. Am J Respir Crit Care Med. 2011;184:1076-84. http://dx.doi.org/10.1164/ rccm.201103-05360C

16. World Health Organization. Report of the Tenth Meeting WHO Strategic and Technical Advisory Group for Tuberculosis (STAGTB), 27-29 September, 2010. Geneva, World Health Organization; 2010. Fecha de consulta: 30 de noviembre de 2015. Disponible en: http://www.who.int/tb/ advisory_bodies/stag_tb_report_2015.pdf?ua=1.

17. Centers for Disease Control and Prevention. Updated guidelines for the use of nucleic acid amplification tests in the diagnosis of tuberculosis. Morb Mortal Wkly Rep. 2009:58:7-10

18. Lawn SD, Zumla Al. Diagnosis of extrapulmonary tuberculosis using the Xpert( $(\AA)$ MTB/RIF assay. Expert Rev Anti Infect Ther. 2012;10:631-5. http://dx.doi.org/10.1586/eri.12.43

19. Scott LE, Beylis N, Nicol M, Nkuna G, Molapo S, Berrie $L$, et al. The diagnostic accuracy of Xpert MTB/RIF on extrapulmonary tuberculosis specimens: Establishing a laboratory testing algorithm for South Africa. J Clin Microbiol. 2014;52:1818-23. http://dx.doi.org/10.1128/JCM.03553-13

20. Tortoli E, Russo C, Piersimoni C, Mazzola E, Dal Monte $\mathrm{P}$, Pascarella $\mathrm{M}$, et al. Clinical validation of Xpert MTB/RIF for the diagnosis of extrapulmonary tuberculosis. Eur Respir J. 2012;40:442-7. http://dx.doi. org/10.1183/09031936.00176311

21. World Health Organization. Automated real-time nucleic acid amplification technology for rapid and simultaneous detection of tuberculosis and rifampicin resistance. Xpert MTB/RIF assay for the diagnosis of pulmonary and extrapulmonary TB in adults and children: Policy update. Geneva: World Health Organization; 2013. Fecha de consulta: 16 de septiembre de 2015. Disponible en: http:// www.ncbi.nlm.nih.gov/books/NBK258608/.

22. Organización Panamericana de la Salud. Manual para el diagnóstico bacteriológico de la tuberculosis. Parte 2, cultivo. Washington, D.C.: OPS; 2008. 
23. Marín DC, Aristizábal BH. Métodos diagnósticos moleculares en tuberculosis. Med UPB. 2013;32:144-50.

24. Vadwai V, Boehme CC, Nabeta P. A new pillar in diagnosis of extrapulmonary tuberculosis? Clin Microbiol. 2011;49:2540-5. http://dx.doi.org/10.1128/JCM.02319-10

25. Montúfar FE, Aguilar-Londoño C, Saldarriaga-Acevedo C, Quiroga-Echeverri A, Montaño B, et al. Características clínicas, factores de riesgo y perfil de susceptibilidad de las infecciones por micobacterias documentadas por cultivo, en un hospital universitario de alta complejidad en Medellín (Colombia). Rev Chil Infectol. 2014;31:735-42. http://dx.doi. org/10.4067/S0716-10182014000600015

26. Centers for Disease Control and Prevention. Guidelines for prevention and treatment of opportunistic infections in HIV-infected adults and adolescents. MMWR Recomm Rep. 2009;58:1-207.

27. Vittor AY, Garland JM, Gilman RH. Molecular diagnosis of TB in the HIV positive population. Ann Glob Health. 2014; 80:476-85. http://dx.doi.org/10.1016/j.aogh.2015.01.001

28. Lawn SD, Brooks SV, Kranzer K, Nicol MP, Whitelaw A, Vogt M, et al. Screening for HIV-associated tuberculosis and rifampicin resistance before antiretroviral therapy using the Xpert MTB/RIF assay: A prospective study. PLoS Med. 2011;8:e1001067. http://dx.doi.org/10.1371/journal. pmed.1001067

29. Pérez-Guzmán C, Vargas MH, Arellano-Macías M del R, Hernández-Cobos S, García-Ituarte AZ, Serna-Vela FJ. Clinical and epidemiological features of extrapulmonary tuberculosis in a high incidence region. Salud Pública Mex. 2014;56:189-96.

30. Hussain SF, Irfan M, Abbasi M, Anwer SS, Davidson S, Haqqee R, et al. Clinical characteristics of 110 miliary tuberculosis patients from a low HIV prevalence country. Int J Tuberc Lung Dis. 2004;8:493-9.

31. Secretaría de Salud de Medellín. Boletín epidemiológico de Medellín. Alcaldía de Medellín; 2014. Fecha de consulta: 30 de noviembre de 2015. Disponible en: https://www.medellin. gov.co/irj/go/km/docs/pccdesign/SubportaldelCiudadano_2/ PlandeDesarrollo_0_19/Publicaciones/Shared\%20Content/ Boletines/Bolet\% $\bar{C} 3 \%$ ADn\%20epidemiol\%C3\%B3gico\%20 4\%20de\%202015\%20-\%20Tuberculosis.pdf.

32. Clemente M, Palacios J, Penedo A, Álvarez M, Velasco Z, Pando A, et al. Accuracy of the Xpert MTB/RIF test for rapid diagnosis of extrapulmonary tuberculosis. Am J Respir Crit Care Med. 2012;185:A4717. http://dx.doi. org/10.1164/ajrccm-conference.2012.185.1

33. Causse M, Ruiz P, Gutiérrez-Aroca JB, Casal M. Comparison of two molecular methods for rapid diagnosis of extrapulmonary tuberculosis. J Clin Microbiol. 2011;49: 3065-7. http://dx.doi.org/10.1128/JCM.00491-11

34. Realpe T, Mejía G, Zapata E, Barón P, Gómez V, Robledo J. Evaluación del desempeño de una prueba molecular comercial para el diagnóstico de tuberculosis y la resistencia a rifampicina en muestras clínicas. Hechos Microbiológicos. 2014;5:41.

35. Moure R, Martín R, Alcaide F. Effectiveness of an integrated real-time PCR method for detection of the Mycobacterium tuberculosis complex in smear-negative extrapulmonary samples in an area of low tuberculosis prevalence. J Clin Microbiol. 2012;50:513-5. http://dx.doi. org/10.1128/JCM. 06467-11
36. Ferreiro L, San José E, Valdés L. Derrame pleural tuberculoso. Arch Bronconeumol. 2014;50:435-43. http:// dx.doi.org/10.1016/j.arbres.2013.07.006

37. Porcel JM, Palma R, Valdés L, Bielsa S, San-José E, Esquerda A. Xpert $₫$ MTB/RIF in pleural fluid for the diagnosis of tuberculosis. Int J Tuberc Lung Dis. 2013;17:1217-9. http://dx.doi.org/10.5588/ijtld.13.0178

38. Friedrich SO, von Groote-Bidlingmaier F, Diacon AH. Xpert MTB/RIF assay for diagnosis of pleural tuberculosis. J Clin Microbiol. 2011;49:4341-2. http://dx.doi.org/10.1128/ JCM.05454-11

39. Rock RB, Olin M, Baker CA, Molitor TW, Peterson PK. Central nervous system tuberculosis: Pathogenesis and clinical aspects. Clin Microbiol Rev. 2008;21:243-61. http:// dx.doi.org/10.1128/CMR.00042-07

40. Katti MK. Pathogenesis, diagnosis, treatment, and outcome aspects of cerebral tuberculosis. Med Sci Monit. 2004;10:RA215-29.

41. Denkinger CM, Schumacher SG, Boehme CC, Dendukuri N, Pai M, Steingart KR. Xpert MTB/RIF assay for the diagnosis of extrapulmonary tuberculosis: A systematic review and meta-analysis. Eur Respir J. 2014;44:435-46. http://dx.doi.org/10.1183/09031936.00007814

42. González-Martín J, García-García JM, Anibarro L, Vidal R, Esteban J, Blanquer R, et al. Documento de consenso sobre diagnóstico, tratamiento y prevención de la tuberculosis. Enferm Infecc Microbiol Clin. 2010;28:e1297-20. http://dx.doi.org/10.1016/j.eimc.2010.02.006

43. Agudelo C, Builes L, Hernández M, Robledo J. Nuevos métodos para el diagnóstico del tuberculosis. latreia. 2008;21:321-32.

44. Marlowe EM, Novak-Weekley SM, Cumpio J, Sharp SE, Momeny MA, Babst A, et al. Evaluation of the Cepheid Xpert MTB/RIF assay for direct detection of Mycobacterium tuberculosis complex in respiratory specimens. J Clin Microbiol. 2011;49:1621-3. http://dx.doi.org/10.1128/JCM. 02214-10

45. Centers for Disease Control and Prevention. Testing for TB infection. 2014. Fecha de consulta: 13 de agosto de 2015. Disponible en: http://www.cdc.gov/ tb/topic/testing/ default.htm

46. Penz E, Boffa J, Roberts DJ, Fisher D, Cooper R, Ronksley PE, et al. Diagnostic accuracy of the $\mathrm{Xpert}^{\circledR}$ MTB/RIF assay for extra-pulmonary tuberculosis: A metaanalysis. Int J Tuberc Lung Dis. 2015;19:278-84. http:// dx.doi.org/10.5588/ijtld.14.0262

47. World Health Organization. Xpert MTB/RIF implementation manual: Technical and operational "how-to"; practical considerations. WHO/HTM/TB/2014.1 Geneva: WHO; 2014. Fecha de consulta: 12 de agosto de 2015. Disponible en: http://who.int/tb/publications/xpert_implem_manual/en/.

48. Ajantha GS, Shetty PC, Kulkarni RD, Biradar U. PCR as a diagnostic tool for extra-pulmonary tuberculosis. J Clin Diagn Res. 2013;7:1012-5. http://dx.doi.org/10.7860/ JCDR/2013/5425.3075

49. World Health Organization. The Global Plan to Stop TB, 2006-2015. Fecha de consulta: 10 de julio de 2013. Disponible en: http://www.who.int/tb/publications/2006/en/ 